\title{
Why non-individuality? \\ A discussion on individuality, identity, and cardinality in the quantum context
}

\author{
Jonas Rafael Becker Arenhart \\ Department of Philosophy \\ Fronteira Sul Federal University \\ Chapecó, SC - Brazil \\ jonas.becker2@gmail.com \\ Décio Krause \\ Department of Philosophy \\ Federal University of Santa Catarina \\ Florianópolis, SC - Brazil \\ deciokrause@gmail.com
}

\begin{abstract}
Recently, in the debate about the ontology of quantum mechanics some authors have defended the view that quantum particles are individuals in a primitive sense, so that individuality should be preferred over non-individuality (the alternative option). Primitive individuality involves two main claims: i) every item is identical with itself and ii) it is distinct from every other item. Non-relativistic quantum mechanics is said to provide positive evidence for that position, since in every situation comprising multiple particles there is a well-defined number of them to begin with, and so they must be distinct from each other. We argue that the link between a well-defined number of items and the relation of identity that is being claimed is not imposed by quantum mechanics, but rather by a metaphysical view. Formal evidence is advanced in favor of the thesis that counting may be performed for items without identity (non-individuals), so that quantum mechanics may not be viewed as endorsing an ontology of individuals.
\end{abstract}

Key-words: quantum mechanics; individuality; non-individuals; counting; identity; quasi-set theory.

\section{Resumo}

Recentemente, no debate sobre a ontologia da mecânica quântica, alguns autores defenderam que as partículas quânticas devem ser vistas como indivíduos em algum sentido primitivo, de modo que a individualidade deve ter preferência sobre a não individualidade (a opção alternativa). A individualidade primitiva envolve duas teses principais: i) todo item é idêntico a si mesmo e ii) é distinto de qualquer outro item. A mecânica quântica não relativista é vista por estes autores como fornecendo evidência positiva em favor desta posição, dado que em todas as situações envolvendo múltiplas partículas há um número bem determinado de itens, de modo que devem ser distintos uns dos outros. Argumentaremos que a alegada ligação entre um número bem definido de itens e a relação de identidade não nos é imposta pela mecânica quântica, mas antes por uma doutrina metafísica. Evidência formal é apresentada em favor da tese de que podemos contar itens sem identidade (não indivíduos), de modo que a mecânica quântica não favorece uma ontologia de indivíduos. 
Palavras chave: mecânica quântica; individualidade; não indivíduos; contagem; identidade; teoria de quaseconjuntos.

\section{Ontological problems in quantum mechanics}

Recent debates about the metaphysical nature of quantum entities present us with three options: one may consider those entities as being individuals, as being nonindividuals, or, as a last resource, forget all talk about particular objects and adopt some form of ontic structural realism (OSR). It seems clear that one of those answers should have better credentials than the others, and so, be preferred and adopted. The main problem with that suggestion, however, concerns the grounds for arguing for preferring one of those answers while leaving the others behind. How can we ground our choice? How are we to decide which is the best option?

Historically, the issue has been treated from a very naturalistic point of view. That is, the choice should be made bearing always in mind what quantum mechanics itself dictates us concerning those matters. In that case, the adoption of a metaphysics of non-individuals seems to have at least historical precedence over the other two options. Really, right from the beginning of the theory it was seen by some of the founding fathers of quantum mechanics that it dealt with items without identity, in the sense of having no individuality. That is, it seemed to follow from the strange statistical behavior of quantum particles that they had no individuality, no identity, and so were a very strange-behaved kind of thing. That view was called the Received View on quantum non-individuality (see French and Krause (2006)).

As is well-known, the Received View was soon discovered to be not the only possible metaphysical view compatible with quantum mechanics. Indeed, it was realized that quantum entities could also be seen as individuals. In this case, the principle of individuality employed should be compatible with quantum indistinguishability; that is, since quantum particles were (sometimes) absolutely indistinguishable -- and that was one of the reasons to believe they were non-individuals at first - the principle of individuality should allow for numerical diversity without a qualitative distinction (in the sense of existing two items but no property discerning them). That is the main reason why such principles are called Transcendental Individuality principles, since they allow the individuation to be performed by something over and above the individual's qualities.

The very idea of a transcendental principle of individuality, however, did not fit very well in the naturalistic picture mentioned above, according to which it is quantum mechanics which should dictate what is the nature of the entities dealt with. Something like a bare particular or a Lockean substratum seems just too mysterious to be favored by scientifically minded philosophers. How could quantum mechanics provide positive support for the idea that quantum particles are individuals according to that sense of individuality? Since transcendental individuality poses by definition that the principle of individuality is not empirically detectable, there seems to be no reason to believe that quantum mechanics would authorize the adoption of such a principle. The best we can claim is that quantum mechanics is compatible with it (see French and Krause (2006), chap. 4 for the details).

Did that mean that non-individuality win the game? Not really. Philosophers started to look for further individuality principles which could be employed in the case of quantum mechanics and which, of course, could be better suited than transcendental individuality to cope with some kind of scientifically informed metaphysics. One such approach was made in a series of papers by Muller, Saunders and Seevinck (see 
Saunders (2006), Muller and Saunders (2008) and Muller and Seevinck (2009)). According to their approach, roughly speaking, quantum entities may be discerned by relations derivable in the Hilbert space formalism of quantum mechanics. Since quantum entities of the same kind are indiscernible in the sense of sharing all their properties, their discernibility comes only through some very restricted kind of relations: those that are symmetric and irreflexive. Symmetry ensures us that there is no privileged order between the items being related and which could be employed to discern the items; irreflexivity ensures us that one item is not related to itself, so that if an irreflexive relation holds, we are sure that there are indeed at least two items being related (in the case of binary relations, of course). Those relations, then, could be employed to ground quantum entities' discernibility and individuality. Discernibility by relations that are symmetric and irreflexive is called weak discernibility.

Discerning and individuating by the use of relations, however, poses its own metaphysical troubles, as is shown in the history of metaphysics. As is known, some philosophers have complaint that relations in general presuppose the items being related, and so they cannot account for the individuality and discernibility of those items. So, not everyone was willing to accept that quantum entities are individuals in the sense of being only weakly discernible (see also the criticism of the notion of weak discernibility in Ladyman and Bigaj (2010) and Krause (2010)). More than that, it has been pointed by Dorato and Morganti (2011) that weak discernibility presupposes yet another form of individuality, more fundamental than the one which weak discernibility could provide us. Really, they have claimed that the whole procedure for deriving in quantum mechanics the weakly discerning relations presupposes that we are able to account for a plurality of particles with a well-defined cardinal. How, they ask, could this be achieved without presupposing individuality?

Dorato and Morganti claim that to grant a well-defined cardinality for any plurality of items is itself the manifestation of individuality. That is, individuality, in some very minimal sense, consists of numerical distinction, every item is numerically distinct from every other item, and self-identity, in the sense that every item is identical with itself. It is individuality in the sense comprised by both these features that Dorato and Morganti claim that quantum entities have, and they say it is primitive. That is, individuality, understood as self-identity and numerical diversity is a primitive feature of quantum particles, not grounded by anything else. What is really interesting is that the naturalistic idea according to which individuality should be somehow endorsed by quantum mechanics also appears here; that is, Dorato and Morganti argue that primitive individuality derives its great plausibility because quantum mechanics itself (in the nonrelativistic formulation) always presents situations comprising a plurality of items with well-defined cardinality.

Is that the end of non-individuality? Not at all. Notice that the claim is that quantum mechanics should favor primitive individuality by the simple fact that it presents us with cases in which a plurality of items should be taken for granted. And individuality in the minimal sense proposed above is needed to account for that feature, right? Not really. Notice that only a well-determined cardinality is being claimed here, and that quantum mechanics says nothing about the other condition, the one that requires every item to be self-identical. It seems we could simply abandon that claim and rest content only with the cardinal condition. Really, if we could grant that items without identity do make sense and may be gathered in collections with a well-defined cardinality, then, it seems, we have a good claim that non-individuality is an even simpler position, assuming less concepts and allowing us to understand the main claims of quantum mechanics. 
In this paper we shall argue that non-individuality may have some kind of preference over individuality when this notion is understood precisely in the minimal sense sketched above. We shall point that even though quantum mechanics by itself does not decide the matter of whether an ontology of individuals or an ontology of nonindividuals should be preferred, there are other kinds of reasoning which may be employed to favor the non-individuality package. The kind of reasoning we are talking about here is generally accepted as good and virtuous reasoning in other situations presenting underdetermination: metaphysical economy and simplicity. That is, nonindividuality may do with less what the individuality option does with more. We shall show that some simple formal assumptions and a reasonable understanding of nonindividuals may be consistent with the idea of a plurality of indistinguishable items with a well-defined cardinal. The requirement that items be self-identical may well be left behind as an ontological excess.

\section{Non-individuality, individuality, cardinality}

As we have been discussing, the idea that some items may be non-individuals appeared naturally in the early developments of quantum mechanics. The theory posed a theoretical framework in which some items, it seemed, had to be non-identifiable and obey some kind of permutation symmetry. That is, roughly speaking, in quantum mechanical systems comprised of many particles, even though we must begin the mathematical description by labeling the particles with natural numbers, those labels play no relevant metaphysical role. The fact that quantum particles are permutation symmetric grant us that permuting those labels will have no physical significance, in the precise sense that the expectation values for a measurement made in the system before the permutation will have the same expectation value as a measurement of the same quantity made after the permutation.

That fact is usually mentioned in connection with the particular statistics obeyed by quantum particles. It seems reasonable to study problems concerning individuality and identity for quantum particles looking at their statistical behavior, since we cannot look at one particle at each time and check its features. What the statistics obeyed by quantum particles revealed was that a permutation of particles' labels does not give rise to a distinct state. That is, particles' labels may be permuted and the state remains exactly the same.

Obviously, not everyone agree with that description of the phenomena. As we mentioned before, Dorato and Morganti ((2011) section 6) have advanced the thesis that a well-defined cardinality is always presupposed by quantum mechanics. That is, we always start with a well-defined number of particles (in the non-relativistic theory), and only then we may go on to develop the usual mathematical model in Hilbert space formalism. Starting with $n$ particles of the same kind (that is, sharing the same intrinsic properties), we attribute a Hilbert space to each of them (the same Hilbert space in this case, in which the particles are indiscernible). The state space for the whole system is furnished by the tensor product of those Hilbert spaces.

Now, Dorato and Morganti point out (loc. sit.), to grant that there will always be some well-defined number of particles, one must also grant individuality. That is, only once we agree that the items in question are already individuals we may be able to say with full meaning that there are $n$ items, even if the items in question are indistinguishable. So, it seems, those items must be individuals, since that is precisely what we do in quantum mechanics. Individuality, here, is seen as involving self-identity 
and numerical distinction, in the sense that everything is identical to itself and distinct from every other thing.

Then, we have both the ingredients discussed briefly in the first section: a metaphysical notion of individuality, and some form of naturalism. According to the naturalistic view in action here, it is quantum mechanics which should furnish the information about the metaphysical nature of quantum particles. According to Dorato and Morganti, that is precisely what is happening: quantum mechanics tells us that there is a well-defined number of items, and that is involved in individuality, mainly, in individuality as a primitive feature of individuals. So, what more should we ask? Why don't we accept primitive individuality for quantum particles?

One could point that the relation between quantum mechanics, well-defined cardinality and individuality is not so direct and obvious. The ties may be weaker than we first thought. First of all, only one feature of individuality as defined by Dorato and Morganti was applied, the idea of well-defined cardinality. Self-identity was not mentioned. Is it because quantum mechanics says nothing about self-identity? But then how could we call quantum mechanics in the help of a doctrine which needs selfidentity as a central notion? In this case, self-identity seems to be a further metaphysical addition, not really needed for quantum mechanics (and not endorsed by it either). However, the friend of individuality could object now: self-identity is really needed, since it is necessary to determine the cardinal number of collections of particles. Without identity there is no sense in claiming that a collection has a well-defined cardinality (see also the arguments in Jantzen (2011), following the same lines). There comes our second point: what is being understood by a cardinal here? Really, of what sense of cardinality are we talking about?

Notice that this is a central question to be answered once our metaphysical thesis about individuality relies (at least indirectly) on a notion such as cardinal number (in the sense that there is a plurality of items comprising a collection). If we take cardinality to mean the usual von Neumann cardinal, which is an initial ordinal, then, no doubt some form of identity and maybe discernibility will be needed. However, if the idea is to preserve quantum indiscernibility, then another notion of cardinal may be employed. Which notion is that? How does that relate to the issue of individuality?

We shall propose that the link between individuality, identity and cardinality is not as strong as it may be thought at first sight. That is, we may abandon the idea that items are self-identical, and even so they may form collections that have a well-defined cardinality. Since such a view is tenable (as we shall see), the link individualitycardinality is broken; non-individuality may walk side-by-side with well-defined cardinality. Since this last fact is all that is needed in the quantum mechanical picture, non-individuality seems to be the simplest assumption for an ontology of quantum mechanics.

In the next section we prepare the formal basis for our understanding of how the notion of cardinality may be dissociated from identity and, hence, from individuality. We formalize the idea of a non-individual, and add to it some set theoretical principles. The result is quasi-set theory, the formal apparatus in which we shall built our definition of cardinality.

\section{Formal aspects of non-individuality}

Now, given those features of non-individuals, we may profit from some rigor in the development of a metaphysical framework to deal with those entities. That is, we may develop in the main lines a formal system designed to deal with non-individuals 
and show that many interesting results may be derived from the simple assumption of non-individuality plus some usual set theoretical tools adapted to deal with them. In that sense, we shall argue, a metaphysics of non-individuals is a very fruitful research program, which should be pursued for its metaphysical plausibility and from the very natural way some features of quantum entities fit inside our framework.

To begin, we develop an informal version of quasi-set theory $Q$ (see the details in French and Krause (2006) chap. 7, French and Krause (2010)). This theory was built with the specific purpose of encoding in a formal system the idea of non-individuals and collections of non-individuals. The first thing that must be clear is that we are employing first-order logic without identity. That is, the whole usual logical apparatus of quantifiers, variables, connectives and the like is available, but identity is not. The next step consists in delimiting the structure of the items dealt with in the theory, the basic entities.

Now, since this is a theory about non-individuals, it seems reasonable to accept that some things are non-individuals. We put a predicate for them: $m$, in the sense that $m(\mathrm{x})$ says that $x$ is a non-individual, or, briefly, an m-atom. We also adopt a predicate for individuals: $M$, such that $M(\mathrm{x})$ means that $x$ is an individual, or, briefly, an M-atom. Our first assumption concerning those items is that nothing is both an individual and a non-individual, and that grants a separation in the intended universe of discourse, in the sense that there are two kinds of things, individuals and non-individuals. Furthermore, we do not postulate the existence of atoms of any kind. It is enough that the theory is consistent with their existence (as in the standard approaches to set theory with Urelemente).

Before we can make the difference between individuals and non-individuals more explicit in the theory, we shall accept that items, be them either m-atoms or Matoms, may form collections, or aggregates. To achieve that aim, we assume as a primitive relation the usual membership $\in$ relation. How is this relation supposed to work, since we have only atoms until now? Easy, we proceed as in usual set theories with atoms: a collection is something that is not an atom. To be more precise, we call the collections quasi-sets (or simply q-sets), and if $x$ is a quasi-set we denote that fact by $Q(\mathrm{x})$. Now, q-sets may have any one of the two kinds of atoms as elements, or also other q-sets. So, there comes the question: what is it that makes a non-individual a nonindividual?

As we mentioned before, non-individuals have some characteristics which we expect to mimic in $Q$. The first of them that we have already obtained is that one may gather non-individuals in collections. That is, there are q-sets of m-atoms. The second feature concerns identity: non-individuals do not have identity. Since in $Q$ the concept of identity may not hold for some entities, it seems we are at the good side. What about individuals? Well, we may define identity for the individuals and grant extensionality for q-sets:

$\mathrm{x}=\mathrm{y}={ }_{\text {Def }}[\mathrm{Q}(\mathrm{x}) \wedge \mathrm{Q}(\mathrm{y}) \wedge \forall \mathrm{z}(\mathrm{z} \in \mathrm{x} \leftrightarrow \mathrm{z} \in \mathrm{y})] \vee[\mathrm{M}(\mathrm{x}) \wedge \mathrm{M}(\mathrm{y}) \wedge \forall \mathrm{z}(\mathrm{x} \in \mathrm{z} \leftrightarrow \mathrm{y} \in \mathrm{z})]$

That is, quasi-sets having the same elements are identical and $\mathrm{M}$-atoms belonging to the same q-sets are identical. Then, identity is granted for the right things, but not for nonindividuals, in the sense that it does not make sense to say that a non-individual is distinct from other non-individual, as well as it is impossible to say that a nonindividual is identical to itself.

Before we go on to build q-sets, there is a further feature of non-individuals as they appear in quantum mechanics to be taken into account: they may be indiscernible. 
To express that fact, we introduce the binary relation symbol $\equiv$, so that $x \equiv y$ means that $x$ is indiscernible from $y$. We agree that m-atoms may be indiscernible, and also grant that for items that are not $\mathrm{m}$-atoms indiscernibility and identity are equivalent. Obviously, for m-atoms that equivalence does not hold, since they cannot be related by identity; in this sense, identity and indiscernibility do not coincide for m-atoms, which may be indistinguishable but not identical. Another sense in which identity differs from indistinguishability concerns their relation to membership. We know that if $x=y$, then, i) for any $z$, if $x \in z$ then also $y \in z$, and also ii) if for any $z$, if $z \in x$ then also $z \in y$. In this sense, identity is said to be compatible with membership. Indistinguishability, however, should not be compatible with membership when we are dealing with m-atoms. Indiscernible m-atoms need not belong to the same q-sets. In that sense, in $Q$ it may happen that $x \equiv y$ and $x \in z$, but that does not imply that necessarily it also happens that $y \in z$.

Having that in our hands, we can now proceed to build quasi-sets. Quasi-set theory encompasses basically the same principles employed to build sets in usual set theory: we have the existence of an empty q-set, unordered pairs, power q-set, the separation schema, union, infinity, regularity and the axiom of choice. The idea is that we may build a quasi-set theoretical hierarchy of q-sets, just like the usual set theoretical hierarchy, but this time with two kinds of atoms.

Some of the collections thus obtained will be of a distinct kind, they will contain only $\mathrm{M}$-atoms or collections of $\mathrm{M}$-atoms in their transitive closure. That is, when we investigate their elements, and the elements of their elements, and so on, we discover that no m-atoms are ever present. Those q-sets having that property we call classical qsets, or just sets for brevity. We denote sets in $Q$ by the predicate $\mathrm{Z}$, so that $\mathrm{Z}(x)$ means that $x$ is a q-set that is also a set. With sets we may build inside $Q$ all of the classical mathematics available in ZFU.

Our worries, however, are not with the classical side of the theory, but rather with the non-classical one. Some of its features, we have been arguing, are such that they accommodate the non-individuals picture. There are two further issues which should be mentioned and which represent very well the differences between a theory comprising only individuals and a theory comprising non-individuals. The first point concerns the formation of unordered pairs. Since some of the items do not enter into the identity relation, we cannot say that a pair $\{x, y\}$ is defined as a collection of the form $\{z: z=x$ or $z=y\}$. That definition would rule out pairs of $\mathrm{m}$-atoms. To fix that, we assume as an axiom that there is a collection $z$ having $x$ and $y$ as elements. With the schema of separation, then, we are able to separate from $z$ a q-set $w$ whose elements are those items belonging to $z$ that are indistinguishable from $x$ or from $y$. Notice that when $x$ and $y$ are not m-atoms, then, since identity and indiscernibility coincide, there is really no difference from what happens in classical set theories, and we denote the pair just like we do in those theories as $\{x, y\}$. However, when at least one of those items is an m-atom, we must consider the possibility of $z$ having more than one element indistinguishable from $x$ or from $y$, nothing prevents that. Then, in the pair $w$ formed by the condition that its elements are those items belonging to $z$ that are indistinguishable from $x$ or from $y$, we may end with more than only $x$ and $y$, that is, with more than two elements (in this explanation, cardinal statements such as "more than two elements" are taken only as an explanation at the metalevel, to be understood intuitively -but see below). Those pairs are denoted by $[x, y]$.

The second main difference concerns ordered pairs. If we are to follow the usual Kuratowski definition of ordered pairs, then the ordered pair $\langle x, y\rangle$ is defined as $[[x]$, $[x, y]]$. As before, in the case those items are not m-atoms, the definition reduces to the 
classical case. However, when those items are m-atoms, some interesting results may be derived. Here we are assuming that $[x]$ and $[x, y]$ were obtained by separation from a qset $z$. If $x$ is indistinguishable from $y$, it is easy to prove that when $\langle x, y\rangle$, then also $\langle y, x\rangle$, That is, indistinguishable m-atoms cannot be ordered. It does not make sense to say that one comes first then the other. So, it follows that we cannot define ordering relations for them.

Now, the relation of that fact with our discussion concerning counting and individuality seems clear. If items are indistinguishable and do not have identity, then we cannot order them. Without order, the collection of items in question cannot have an ordinal, in the sense that there is a first element, a second, and so on. Without ordinals, there is no cardinal in the von Neumann sense, according to which cardinals are a special kind of ordinals, known as initial ordinals. So, should we conclude that nonindividuals cannot be elements of collections with a well-defined cardinal? The answer is yes only if the concept of cardinal is defined in the von Neumann style (or any other style which makes essential use of the identity of the entities in question). However, nothing is said about distinct definitions of cardinality, for which those assumptions are not needed. In the next section we shall show how to make sense of the claim that there is a plurality of non-individuals by using the conceptual tools of quasi-set theory. We shall show that cardinality may be defined without identity being defined for everything.

Before we proceed, however, there are two further notions of $Q$ which must be introduced: the notion of a relation and the concept of a quasi-function. A binary relation is as usual a collection of ordered pairs, but recall that ordered pairs are being understood in the weaker sense mentioned above. For a quasi-function, some modifications in the usual concept of function must be provided. The intuitive idea here is that once we pick a domain and an item $x$ in it, there is an image $y$ in the image q-set. Also, every other item in the domain which is indistinguishable from $x$ should be attributed an image indistinguishable from $y$. That is, a quasi-function maps indistinguishable items into indistinguishable images. More formally, $\mathrm{f}$ is a quasifunction from $\mathrm{A}$ to $\mathrm{B}$ if and only if $\mathrm{f}$ is a quasi-relation between $\mathrm{A}$ and $\mathrm{B}$ such that for every $x \in \mathrm{A}$ there is a $y \in \mathrm{B}$ such that $\langle x, y>\in \mathrm{f}$, and if $\langle w, z>\in \mathrm{f}$ and $x \equiv w$, then $y \equiv z$. Notice once again that when none of the items involved is a m-atom, then the definition collapses in the classical case.

Now, we proceed by showing how a notion of cardinality may be derived without presupposing the identity of the items being counted.

\section{Counting, Cardinality and Non-Individuality}

In this section we analyze the relationships between three concepts: counting, cardinality and non-individuality. Firstly, it may be simply stated that they are very closely related: to attribute cardinality to some collection, one must be able to count the items in it. Counting, on the other hand, is a kind of activity; one attributes numerals in an increasing order to the members of the collection. Obviously, the numerals must be uniquely attributed, so that each item is labeled only once with a numeral. Of course one can only do that to items having well-defined identity conditions, items able to bear labels, and able to be individuated. So, the obvious conclusion would be that only individuals can be counted, can form collections with cardinality, and so on (that is basically the idea exposed in Jantzen (2011), and seems to be accepted also by Dorato and Morganti (2011)). 
Now, if that kind of reasoning is right, then the non-individuals metaphysical package for quantum particles is in trouble. Really, under those circumstances, that view will not be able to explain the numerical difference of particles appearing in quantum mechanics, not even the simple fact that in this theory we deal with systems comprising multiple particles. So, how should we understand those notions in the context of a metaphysics of non-individuals? Can we make sure that identity does not sneaks in unnoticed in our conceptual framework when we apply the notion of cardinal? That is what we shall go into now.

The first point to be noticed is that the relation counting-cardinality is not something one should take for granted. First of all, even in classical set theory, such as Zermelo-Fraenkel, one can define cardinal numbers in such a way as to be completely divorced from a corresponding ordinal, for in fact, the definition making those links, the von Neumann definition, is only one among many different options. That shows that one does not need to link those notions so strictly. In the second place, counting is one of the notions that most philosophers have taken as linked with a principle of individuation without much analysis, so that one should at least try to take a critical stance in relation to it before conclusions are drawn. Our point here will be that even though the usual understanding of counting does seem to presuppose some kind of individuality, one does not need to be concerned with it, for alternative counting procedures may be used that do not rely on the identity of the counted items.

A first proposal to overcome the difficulties may be seen explicitly in the usual presentations of quasi-set theory (see French and Krause (2006), chap. 7). When it comes to deal with cardinalities, in the usual approaches to the theory, it is the common strategy to deal with cardinalities simply to adopt as a primitive term the weaker notion of quasi-cardinals. The point behind this axiomatic approach is that even though one cannot attribute ordinals to collections of m-atoms (since we cannot define order relations in them), one may still attribute them cardinals. That we do through the quasicardinal axioms; we impose by force of axioms that every quasi-set has a cardinal, but not every quasi-set has an associated ordinal. Here, obviously, when we say that a q-set does not have an associated ordinal we mean that it is not associated in the usual way, through a bijection, to an ordinal. By the very fact that cardinals are also ordinals, every q-set having a cardinal does also have an ordinal, but that ordinal plays no role in ordering or labeling the elements of the q-set. If one does not feel comfortable with cardinals attributed by axioms, it is enough to remember that this is not a privilege of quasi-set theory, for sometimes one introduces cardinal in classical theories by means of axioms.

This first solution may be seen as a sharpening of proposal made by many authors, for example, Toraldo di Francia (1998). According to that proposal, one can keep both the non-individuality and the cardinality for quantum particles, but ordinals, understood as attributing an order to the elements of a collection of non-individuals must be avoided. In fact, that same idea may be seen as encapsulated in claims made by Redhead and Teller (see (1991), (1992)). According to those authors, the very possibility of labeling things, as one apparently needs to do in the usual counting notion, does in fact imply some principle of individuality for the particles, something they have called, not surprisingly, Label Transcendental Individuality. Since they also take particles to be indiscernible and non-individuals, instead of proposing that the notion of cardinal be taken as primitive, they propose a shift to the Fock space formalism, in which one keeps only the number of particles in each situation, but no labels or counting would remain (but see French and Krause (2006) for a criticism of the idea that a Fock space formalism solves the problem). 
Obviously, those proposals have their own virtues and difficulties. In the first case, in attributing cardinality by axioms, one may complain that this solves the problem but does not explain why collections of non-individuals have a cardinal. They simply have it, as a brute fact (some people, of course, see it as a virtue). In the proposal to shift to a Fock space formalism, the difficulty is that the very mathematical construction of the formalism is made through the use of Hilbert spaces, which on their turn use the forbidden notions of labeling and counting the particles. So, even though defensible, one may also want to look for an alternative solution.

A third proposal, one we shall investigate here, concerns the possibility of defining cardinality for finite (for this moment understood only in the intuitive sense but see below) collections of non-individuals. Obviously, we employ the framework of quasi-set theory to do exactly that. The idea is that one may define cardinality through some kind of non-standard definition of counting, doing all that in such a way that identity is not needed for the items being counted. One such proposal was made, as we already mentioned, by Domenech and Holik (see (2007)). Their proposal consists of a simple idea: to know how many elements there are in a collection without having to identify or label them, one only needs to devise a procedure through which one can eliminate the members of the collection one by one, successively, so that after some finite number of applications of the procedure one obtains an empty collection. The number of elements in the collection is the number of times we applied the procedure to make the collection empty. Of course, that was only a metamathematical intuitive description of the process, which, when defined in $Q$ does not work in a succession of steps taken as a succession in time.

So, there are two basic problems to be solved if the idea works. First of all, one must make clear what are those units that are to be extracted from the collection and how we can be sure that they indeed have the desired properties of being 'one' item. That must be characterized without appeal to the notion of cardinal, obviously, and so, without making the one-to-one elimination a process of individuation of the entities. Granted that, we can be sure that, intuitively speaking, one is always taking one element from the collection in case. The second point to be taken care of concerns the extracting procedure. It must be furnished in such a way that one does not make appeal to the identity of the elements being extracted, mainly because the entities of the collections we want to count have no identity. It is important to mention that our process of "elimination" of the elements of a q-set act in a very similar way as a process of ionization in chemistry. We want our definition to mirror that idea in the formal system. Of course someone can say that, in eliminating one element, we necessarily individuate it. This makes sense from the intuitive point of view. But, in the process of ionization, suppose that an electron is eliminated from the outer shell of a certain atom. This electron merges with others in the laboratory, so that there will be no more sense in saying which electron is that one. Its supposed identity is lost forever; thus, how can we regard it as an individual? The same happens here; the alleged individuality of the eliminated element shows only that it can be though of as a "something", an entity of some kind, but of course not as having the standard properties we ascribe to individuals, and we do not need to think of it as having self-identity. So, the process may be performed without having to assume or presuppose that the items in question are individuals.

Satisfied both conditions above, one needs only to put the machinery to work in such a way that the extracting procedure takes one element at each time (metaphorically speaking), and that once it reaches the empty set we have somehow recorder the number of times we have applied the procedure, for that will give us our cardinal. 
Now, that idea, despite its abstract appearance, may be seen as mirroring some kind of laboratory practice. In fact, one can think about situations in laboratory where we can employ a specific procedure to count the elements of a collection without being able to identify them. For an example of such procedure encapsulating the features of the counting notion proposed by Domenech and Holik, consider the case in which we wish to count how many electrons an Helium atom has. One possibility consists in putting the atom in a cloud chamber and ionizing it with radiation; the result will be one track of an ion and one of an electron. Repeating the procedure, we would once again see the track of one ion and one electron. Now, after the second stage we cannot extract more electrons, and the process ends in two steps, so that we know that the atom had two electrons. Notice that it does not matter which electron was the first and which one was the second, we cannot identify them, although we can determine how many of them there are (see Domenech and Holik (2007), p. 867).

We believe that both features of the counting process thus proposed can be made clear in the framework of quasi-set theory, with the definition we shall propose now. This is not the definition advanced by Domenech and Holik themselves, but a simpler one (see Arenhart for more discussion (2011). It is a virtue of definitions like this one to make clear the conceptual separation between identity, counting, and cardinality. The conceptual frameworks involving non-individuals benefit from such technical devices, since they make clear some presuppositions that need not be assumed, like the one linking identity, cardinals, and counting. Let us check the definition.

\subsection{Finite quasi-cardinals in $Q$}

Now, let us see more closely a definition of finite quasi-cardinals in $Q$, a definition which, as we mentioned before, is simpler than the one presented by Domenech and Holik, although it relies on the same guidelines proposed by those authors. For convenience only, instead of 'quasi-cardinal' we shall use throughout this section the simpler word 'cardinal'. We shall not give all the details, since that would take us very far, but we give all the relevant definitions. The motivation for our definition, as we mentioned before, is very simple, and aims at reflecting some kind of laboratory practice: we can make a finite q-set empty simply by taking out its elements one at a time. We can then count the number of steps required for this process to end and put as a reasonable definition that this is the number of elements of the q-set, i.e., its cardinal (once again, recall that this is a heuristic explanation provided in the metalanguage). As we have discussed, this process does not make use of the identity of the elements being taken out, and so, it is suitable for collections of m-atoms. First of all, we formulate the axiom of choice, since we will need it:

(AC) If $\mathrm{A}$ is a q-set whose elements are non-empty q-sets, then there is a q-function $\mathrm{f}$ such that for every $B$ belonging to $A, f(B) \in B$.

The next important step comes from the fact that our procedure applies to finite q-sets only. This could be rendered impossible if the only definition of finiteness were the usual one, according to which a collection is finite if and only if there is a one to one correspondence between that collection and some natural number. The point here is that this definition of finiteness comprises also the very idea of counting we wish to avoid, the idea that one can label every element of the collection being counted. So, for the concept of a finite collection we employ the Tarskian definition of finiteness, one that 
does not suffer from those difficulties. Before stating that definition, we need the notion of a $\subset$-minimal element (where $\subset$ is proper inclusion):

Given a q-set $\mathrm{B}$ whose elements are q-sets, we call an element $\mathrm{A}$ of $\mathrm{B} \subset$-minimal if $\forall \mathrm{C}(\mathrm{C} \in \mathrm{B} \rightarrow \neg \mathrm{C} \subset \mathrm{A})$.

Given that, the Tarski finite q-sets are defined as follows:

A q-set A is finite in the sense of Tarski if every non-empty collection of subsets of A has a $\subset$-minimal element.

In the following, finite q-sets shall always mean Tarski-finite q-sets, unless otherwise stated. The next step is the definition of the strong singleton of an item A. As we mentioned before, one can introduce in $Q$ the idea of a collection having intuitively only one element indiscernible from certain other thing, an m-atom, for instance. That definition may be introduced with the help of the notion of quasi-cardinal, when it is a primitive symbol of the theory, or it may alternatively be introduced by a definition not using that notion. Here, obviously, we must follow the second approach, since we do not have quasi-cardinals as primitive in our theory. We will denote by $<\mathrm{A}>$ the strong singleton of A (as in French and Krause (2006) p. 293).

i) If $\mathrm{A} \in \mathrm{B}$, we define $\mathrm{S}_{\mathrm{A}}$ by $[\mathrm{s} \in \wp([\mathrm{B}]): \mathrm{A} \in \mathrm{s}]$;

ii) $\angle \mathrm{A}>=_{\text {Def }} \cap \mathrm{T} \in \mathrm{Sx} \mathrm{T}$

The notion of strong singleton will play the role of unit we discussed before. Really, since our plan is to eliminate the elements of a collection one by one, we already have what are the things to be counted intuitively as one: strong singletons of elements from the collection. As we have already commented, the strong singleton of A is a q-set containing what we would intuitively take as only one element indistinguishable from A. The point of whether the strong singleton have the right to claim to be a collection with only one element, since we do not have yet a notion of quasi-cardinal, was settled by Domenech and Holik. These authors have proved that the strong singleton has a property that only collections with one element should intuitively have: their only subsets are themselves and the empty q-set (see their (2007) p. 865). Or more clearly stated: for any $\langle x\rangle$, if $y \subseteq\langle x\rangle$, then either $y$ is the empty q-set or $y$ is $\langle x\rangle$ itself. So, it seems that we are done with the first difficulty mentioned above, that is, we have stipulated what is to count as a unity to be eliminated from every q-set in the counting procedure.

Next, we must provide some procedure to extract strong singletons from any given collection. We do that through a quasi-function which, intuitively speaking, takes elements out of a q-set, one by one. We call this quasi-function subtraction function. Given a finite q-set $\mathrm{A}$, by (AC) there is a choice function $g$ for $\wp(\mathrm{A}) \backslash\{\varnothing\}$, where " " denotes the difference operation between q-sets. Then, we define the subtraction function $h$ from $\wp(\mathrm{A})$ to $\wp(\mathrm{A})$ :

1) If $\mathrm{B} \neq \varnothing$, then $\mathrm{h}(\mathrm{B})=\mathrm{B} \backslash \mathrm{g}(\mathrm{B})>$;

2) If $\mathrm{B}=\varnothing$, then $\mathrm{h}(\mathrm{B})=\varnothing$. 
The idea behind this definition is that $h$ picks an element from each subset of $\mathrm{A}$, extract it, and gives us back still another q-set, call it A', which is a subset of A, and intuitively speaking, we should understand that the resulting subset $A^{\prime}$ ' has one element less than A. Applying the function to the empty q-set results in the empty q-set again. Obviously, the motivation behind our proposal demands that we do not stop with A' unless it is empty. So, we must explain now how we can iterate the extracting process. We do that through an application of the recursion theorem, which is a theorem of $Q$. We define by recursion a quasi-function $f$ from the q-set of natural numbers to $\wp(\mathrm{A})$ :

1. $\mathrm{f}(0)=\mathrm{A}$;

2. $f(n+1)=h(f(n))$.

So, there we have it. That grants us a way to iterate the extraction process and allow us to arrive, in some point in the process, in the empty q-set. Really, we can be sure that the process will arrive at the empty q-set because A is a Tarski-finite q-set. The point can be proved this way: suppose that the quasi-function $\mathrm{f}$ defined above did not arrive at the empty q-set for some natural number $\mathrm{n}$. Then, in this case we would have provided a family of non-empty q-sets that does not contain a $\subset$-minimal element, violating the hypothesis that $\mathrm{A}$ is Tarski finite. So, any finite q-set will have only a finite number of stages in the process of 'eliminating' its elements and coming to be empty. As we have already explained, we may reasonably define the cardinal of a finite q-set as the least number in which we have reached the empty q-set, that is, in which the collection is empty:

The cardinal of $A$, denoted qc(A), is the least natural number $n$ such that $\mathrm{f}(\mathrm{n})=\varnothing$.

For that definition to make perfect sense, one must show that the cardinal of a finite q-set is unique, so that the introduction of the symbol $q c$ may be justified. We shall not go into that here, mentioning only that it can be done (see the relevant proofs in Arenhart (2012)). Notice that we have made use of no further axiom to introduce the notion of cardinal, and then, if all we are interested in are finite collections, then we may confidently begin with $Q$ without any notion of quasi-cardinal as a primitive concept. Obviously, from that definition we could go on and establish other interesting results, as for example the one granting us that for objects satisfying $\mathrm{Z}$, the cardinal as defined above coincides with the usual cardinal, defined through the ordinals. Then, our definition preserves the ideas already introduced by the classical definition of counting, and extends the notion of cardinal to collections of objects without identity.

Now, that seems to be a point in favor of a conceptual framework in which some of the entities do not have identity. Really, since much of what is done with identity can be done without it, then, it seems, we can confidently claim that no trouble shall be faced on that front. Our point here is that the claims linking identity, counting and cardinality are not definitive against an ontology of non-individuals, since, as we have showed, alternative notions may be rigorously presented which do not presuppose the ties between those concepts. This is a typical case in which, if you want to show something works, then, show how to do it.

\section{Conclusion}

Now, it seems, we are in a better position to answer to the question which is the title of this paper, that is, why non-individuality? We have seen that the quantum 
formalism employs somehow the fact that items may come in pluralities. We label those items in order to be able to furnish the mathematical tools that allow us to formulate the theory and deal with the physical system. Does that pose in itself any special metaphysical constraint on the nature of quantum particles? Dorato and Morganti (2011), as well as Jantzen (2011) think it does. According to those authors, the fact that there is a well-defined number of entities is a good evidence to grant that those items are individuals. Without identity for those items there would not be any sense in saying that there is a specific number of them.

So, quantum mechanics was seen as furnishing the needed support for a metaphysics of individuals, in which individuality was taken as a primitive ungrounded notion. There are two relevant aspects to that claim. First of all, quantum mechanics grants the support to the claim that there is a precise number of items in every occasion. Second, there is the metaphysical claim that it only makes sense to say that a situation comprises a precise number of items when those items are individuals. Since almost every other attempt to ground quantum particles' individuality has its weaknesses, individuality must be better taken as primitive, in the sense that individuals are selfidentical and numerically distinct from every other item. Then, putting both together, quantum mechanics favors a metaphysics of individuals, in which individuality is understood as primitive.

We have argued that the link between what we get from quantum mechanics and individuality has a weak link. In fact, we may point to it precisely in the metaphysical part of the argument in the above paragraph: it is not necessary that items be individuals (or be self-identical) for a well-defined number of them in every situation. As we have seen, it makes perfect sense to attribute cardinality to collections of items for which identity does not make sense, the non-individuals. So, from the point of view of metaphysical coherence, there is nothing wrong with a metaphysics of non-individuals. Also, it does the same job without assuming identity.

From a naturalistic point of view, we see now, there is no compelling evidence that primitive individuality should be accepted on quantum mechanical grounds. The acceptance of that position requires that we link the stipulation of a cardinal number to any collection of quantum particles with individuality. However, that is, as we mentioned, this is a metaphysical assumption, and not something to be derived with necessity from quantum mechanics. As far as quantum theory goes, non-individuals are all we need and perhaps constitute the best metaphysics available.

References

ARENHART, J. R. B. - "A discussion on finite quasi-cardinals in quasi-set theory". In Foundations of Physics, 41, 2011, pp. 1338-1354.

ARENHART, J. R. B. - "Finite Cardinals in Quasi-set Theory". In Forthcoming in Studia Logica, 2012.

DOMENECH, G., and HOLIK, F. - "A discussion on particle number and quantum indistinguishability". In Foundations of Physics 37(6), 2007, pp. 855-878.

DORATO, M. and MORGANTI, M. - "Grades of Individuality. A pluralistic view of identity in quantum mechanics and in the sciences". Forthcoming in Philosophical Studies 2011. 
FRENCH, S. and KRAUSE, D. - Identity in Physics. A historical, philosophical and formal analysis. Oxford: Oxford University Press, 2006.

FRENCH, S., and KRAUSE, D. - "Remarks on the theory of quasi-sets". In Studia Logica, 95(1-2), 2010, pp. 101-124.

JANTZEN, B. C. - "No two entities without identity". In Synthese, 181(3), 2011, pp. 433-450.

KRAUSE, D. - "Logical aspects of quantum (non-)individuality". In Foundations of Science, 15(1), 2010, pp. 79-94.

LADYMAN, J - "Structural Realism". The Stanford Encyclopedia of Philosophy (Summer 2009 Edition), Edward N. Zalta (ed.), May 22, 2009, [retrieved 11/03/2012]. Available in http://plato.stanford.edu/archives/sum2009/entries/structural-realism/.

LADYMAN, J., and BIGAJ, T. - "The Principle of the Identity of Indiscernibles and Quantum Mechanics”. In Philosophy of Science, 77, 2010, pp. 117-136.

MULLER, F.A., and SAUNDERS, S. - "Discerning Fermions". In British Journal for the Philosophy of Science, 59, 2008, pp. 499-548.

MULLER, F.A., and SEEVINCK, M.P. - "Discerning Elementary Particles". In Philosophy of Science. 76(2), 2009, pp. 179-200.

REDHEAD, M. L. G., and TELLER, P. - "Particles, Particles Labels, and Quanta: The Toll of Unacknowledged Metaphysics". In Foundations of Physics, 21(1), 1991, pp. 4362 .

REDHEAD, M. L. G., and TELLER, P. - "Quantum Physics and the Identity of Indiscernibles". In British Journal for the Philosophy of Science, 43, 1992 pp. 201-218.

SAUNDERS, S. - “Are Quantum Particles Objects?”. In Analysis 66, 2006, pp. 52-63.

TORALDO DI FRANCIA, G. - A world of individual objects? In CASTELLANI, E. (ed.) - Interpreting Bodies: Classical and Quantum Objects in Modern Physics. Princeton: Princeton Un. Press, 1998, pp. 21-29. 